\title{
Selective production of red azaphilone pigments in a Monascus purpureus mppDEG deletion mutant
}

\author{
Bijinu Balakrishnan ${ }^{1}$ (D) $\cdot$ Yoon Ji Lim ${ }^{1} \cdot$ Seok Hyun Hwang ${ }^{1} \cdot$ Doh Won Lee ${ }^{1}$ \\ - Si-Hyung Park ${ }^{2} \cdot$ Hyung-Jin Kwon ${ }^{1}$ (CD
}

Received: 3 August 2017 / Accepted: 25 August 2017 / Published Online: 30 September 2017

(C) The Korean Society for Applied Biological Chemistry 2017

\begin{abstract}
The Monascus azaphilone (MAz) pigment is a wellknown food colorant that has yellow, orange and red components. The structures of the yellow and orange MAz differ by two hydride reductions, with yellow MAz being the reduced form. Orange MAz can be non-enzymatically converted to red MAz in the presence of amine derivatives. It was previously demonstrated that $m p p E$ and $m p p G$ are involved in the biosynthesis of yellow and orange MAz, respectively. However, $\triangle m p p E$ and $\triangle m p p G$ knockout mutants maintained residual production of yellow and orange MAz, respectively. In this study, we deleted the region encompassing $m p p D, m p p E$ and $m p p G$ in $M$. purpureus and compared the phenotype of the resulting mutant ( $\triangle m p p D E G)$ with that of an $m p p D$ knockout mutant $(\triangle m p p D)$. It was previously reported that the $\triangle m p p D$ strain retained the ability to produce MAz but at approximately $10 \%$ of the level observed in the wildtype strain. A chemical analysis demonstrated that the $\triangle m p p D E G$ strain was still capable of producing both yellow and orange $\mathrm{MAz}$, suggesting the presence of minor $\mathrm{MAz}$ route(s) not involving $m p p E$ or $m p p G$. Unexpectedly, the $\triangle m p p D E G$ strain was observed to accumulate fast-eluting pigments in a reverse phase high-performance liquid chromatography analysis. A LCMS analysis identified these pigments as ethanolamine derivatives of red MAz, which had been previously identified in an $m p p E$ knockout mutant that produces high amounts of orange MAz.
\end{abstract}

Hyung-Jin Kwon $(\triangle)$

E-mail:hjink@mju.ac.kr

${ }^{1}$ Department of Biological Sciences and Bioinformatics, Myongji University, Yongin-si, Gyunggi-do 17058, Republic of Korea

${ }^{2}$ Department of Oriental Medicine Resources and Institute for Traditional Korean Medicine Industry, Mokpo National University, Muan-gun, Jeollanam-do 58554, Republic of Korea

This is an Open Access article distributed under the terms of the Creative Commons Attribution Non-Commercial License (http://creativecommons. org/licenses/by-nc/3.0/) which permits unrestricted non-commercial use, distribution, and reproduction in any medium, provided the original work is properly cited.
Although the underlying mechanism is largely unknown, this study has yielded an $M$. purpureus strain that selectively accumulates red MAz.

Keywords Azaphilone pigment · Monascus purpureus Phenotypic characterization of mppDEG deletion mutant Selective production of red azaphilone pigments

\section{Introduction}

Monascus species, such as Monascus ruber, Monascus pilosus, and Monascus purpureus, among others, are filamentous ascomycetes fungi that have been widely used in food fermentation in East Asia (Chen et al. 2015). The statin function of monacolin $\mathrm{K}$ is well known with respect to the bioactivity of Monascus-fermented products, (Nguyen et al. 2017). Additional renowned secondary metabolites of Monascus are a series of polyketide pigments that belong to azaphilone class (Feng et al. 2012; Patakova 2013). Azaphilone (Az) compounds are a group of fungal aromatic polyketides featuring an oxidized pyranoquinone bicyclic structure with a tertiary alcohol that is generally acylated. Az compounds display diverse biological activities that apparently involve interfering with specific protein-protein interactions (Gao et al. 2013). Az is synthesized by a non-reducing fungal polyketide synthase with a reductive release domain (NR-fPKS-R) and its collaborating enzymes (Zabala et al. 2012). The following chemical modifications generate structural diversity in Az compounds (Winter et al. 2012; Balakrishnan et al. 2013).

Monascus Az (MAz) contains yellow (monascin, Y-1, and ankaflavin, Y-2), orange (rubropunctatin, O-1, and monascorubrin, O-2) and red pigments (Fig. 1). Yellow MAz contains a reduced pyranoquinone core, and has a visible absorption peak at $390 \mathrm{~nm}$. Orange MAz, bearing a typical Az pyranoquinone core, has a maximum absorption at $470 \mathrm{~nm}$ and is converted into red MAz in 
the presence of amine (Balakrishnan et al. 2013). A characteristic absorption peak of red MAz can be found at approximately 410 and $500 \mathrm{~nm}$ (Feng et al. 2012; Shi et al. 2016). The pH dependency of the visible absorption spectra of MAz has been documented (Shi et al. 2016). The term Az was coined from a tendency of incorporating a nitrogen atom to generate a vinylogous g-pyridone moiety in red MAz. However, many Az members, including yellow MAz, have no affinity for nitrogen. Regarding to bioactivities, health benefit activities of yellow MAz were extensively studied (Lin et al. 2017).

We previously identified MAz biosynthetic gene clusters in Monascus pilosus (Balakrishnan et al. 2013) and M. purpureus (Balakrishnan et al. 2014a) (Fig. 2). Subsequent gene knockout studies in $M$. purpureus led us to the isolation of MAz biosynthetic intermediates, helping us to elucidate the biosynthetic pathway (Balakrishnan et al. 2013; 2014a; 2014b; Bijinu et al. 2014; Balakrishnan et al. 2017a; 2017b) (Fig. 1). The MpPKS5 gene encodes NR-fPKS-R, which is required for MAz biosynthesis, and its activity is supported by $\mathrm{MppD}$, a serine hydrolase homolog (Balakrishnan et al. 2015). A knockout of $m p p D$ did not abolish MAz production but lowered the MAz levels to $10 \%$ of that observed in the wild-type strain. The participation of an MppD- like protein was also demonstrated in citrinin biosynthesis, but the biochemical role of these serine hydrolase homologs is not yet understood (Balakrishnan et al. 2016; He and Cox 2016). The MpPKS5 product is converted into azanigerone E by MppA and MppF (Bijinu et al. 2014). The acyltransferase MppB then decorates the resulting intermediate with a 3-oxo-acyl moiety that is generated by MpFAS2 (Balakrishnan et al. 2014b). It has also been proposed that subsequent modifications by Mpp7 and MppC generate a hypothetical intermediate (MAz-INT) that then enters into either a reductive (yellow MAz) or an oxidative (orange MAz) pathway (Balakrishnan et al. 2014a; Bijinu et al. 2014) (Fig. 1). It was also demonstrated that the $m p p E$ and $m p p G$ products control the reductive and oxidative pathways, respectively (Balakrishnan et al. 2017a; 2017b). In detail, a significant impairment of yellow MAz production was observed in a gene inactivation mutant of $m p p E(\triangle m p p E)$, as was orange $\mathrm{MAz}$ production in an $m p p G$ inactivation mutant $(\triangle m p p G)$. However, both the $\triangle m p p E$ and $\triangle m p p G$ mutants are still able to produce yellow and orange MAz, respectively.

To examine whether each oxidoreductase gene ( $m p p E$ and $m p p G$ ) could partially complement the absence of the other gene, we tried to generate a double mutant that was inactivated for both

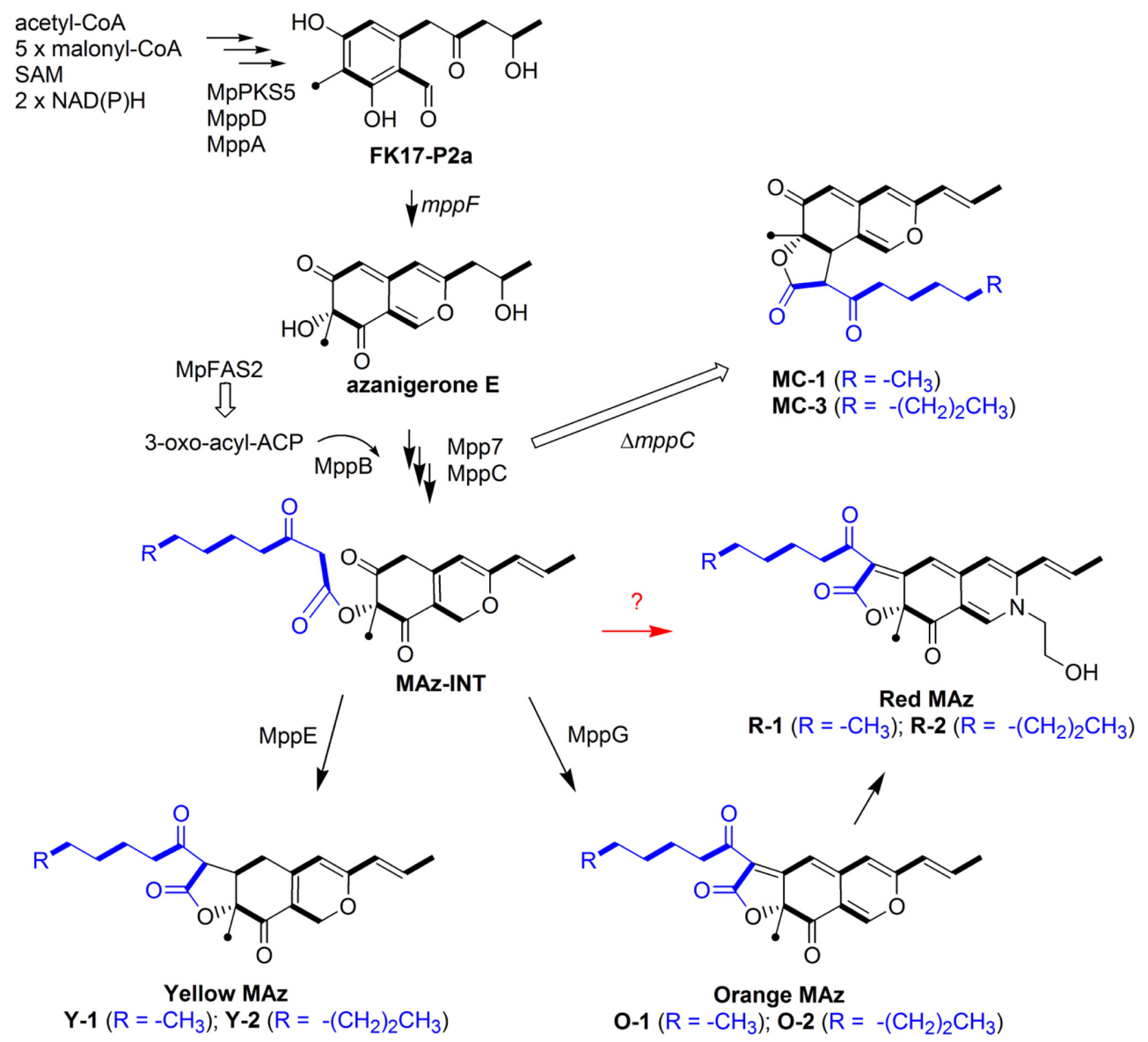

Fig. 1 Structures of MAz compounds with their proposed biosynthetic pathway. To emphasize the biosynthetic origin, the acetate units and $S$-adenosylL-methionine-derived carbons are denoted with bars and black dots, respectively. The compound names are in bold 
Table 1 Primers used in this study. The nucleotide sequences introduced for In-Fusion cloning are underlined and italiczed. The sequence information was retrieved from the $M$. purpureus genome sequence portal at the Department of Energy-Joint Genome Institute (http:// genome.jgi.doe.gov/Monpu1/Monpu1.home.html)

\begin{tabular}{ll}
\hline \hline \multicolumn{1}{c}{ Name } & \multicolumn{1}{c}{ Sequence $\left(5^{\prime} \rightarrow 3^{\prime}\right)$} \\
\hline hyg-F & TGAAATACGTCGAGCCTGCT \\
Hyg-mppE-R & CTGGCTCAAATACCCCCTGTGCATTCTGGGTAAAC \\
MppEdw-F & GGGTATTTGAGCCAGGTGAA \\
MppEdw-R & $\underline{\text { TACCGAGCTCGAATTCGACCAGGCACTGGATGATCT }}$ \\
MppG-up-F & CCATGATTACGAATTCAAGGGGACGTTGATGTCTTG \\
MppG-up-R & $\underline{\text { GCTCGACGTATTTCAATAAGGACATCGCGGTTGAC }}$ \\
\hline
\end{tabular}

mppE and $m p p G$. However, our attempt to generate the double mutant was unsuccessful. As an alternative approach, we deleted a region compassing $m p p E, m p p G$ and $m p p D$, with $m p p D$ being located between $m p p E$ and $m p p G$. We investigated the MAz profile of the resulting mutant $(\triangle m p p D E G)$ compared with an $m p p D$ knockout mutant $(\triangle m p p D)$. The $\triangle m p p D E G$ mutant remained capable of producing yellow and orange MAz, suggesting that there are unidentified, minor routes for MAz biosynthesis. Interestingly, the $\triangle m p p D E G$ deletion resulted in an accumulation of red MAz derivatives, which is the primary finding of this study. We discussed the possible mechanisms how $\triangle m p p D E G$ accumulate these red MAz derivatives while producing a residual level of orange MAz.

\section{Materials and Methods}

Strains, culture conditions, and extraction methods

Monascus purpureus KACC (Korean Agricultural Culture Collection) 42430 and Agrobacterium tumefaciens AGL1 were used in this study. M. purpureus and its derivatives were maintained on potato dextrose agar (PDA) for 7 days at $30{ }^{\circ} \mathrm{C}$. The volume of PDA in each plate was approximately $50 \mathrm{~mL}$. Five $1-\mathrm{cm}^{3}$ PDA blocks inoculated with $M$. purpureus were used to initiate potato dextrose broth cultures (PDB; Gellix ${ }^{\mathrm{TM}}$, Ventech Bio, Seoul, Korea). This PDB brand affords a high level of orange MAz production from the M. purpureus strain used in this study. The PDA culture was extracted with a chloroform and methanol mixture (v/v 1:2). For liquid cultures, mycelia were separated by centrifugation at $6,000 \mathrm{rpm}$ at $4{ }^{\circ} \mathrm{C}$ for $15 \mathrm{~min}$, and the collected mycelia pellets were also extracted with a chloroform and methanol mixture (v/v 1:2). The phase separation was facilitated by the addition of water, and the chloroform layer was reserved and evaporated under reduced pressure. The extracts were finally dissolved in $1 \mathrm{~mL}$ of methanol and used for chemical analyses. The organic extract from each $50 \mathrm{~mL}$ culture was dissolved in 0.5 $\mathrm{mL}$ of methanol and was used in high-performance liquid chromatography (HPLC) analyses. The two microliter methanol samples used for injection are denoted as $1 \mathrm{x}$ in this study.

\section{Genetic knockouts of $m p p D, m p p E$ and $m p p G$}

The primers used in this study are listed in Table 1. Polymerase chain reaction (PCR) was performed with Herculase II Fusion DNA Polymerase (Agilent, Santa Clara, CA, USA). To generate an mppDEG inactivation construct, 1,856 and 1,812 bp DNA fragments upstream and downstream of the $m p p D E G$ region were PCR amplified with the primer pairs of MppGup-F/MppGup-R and MppEdw-F/MppGdw-R, respectively. These primer pairs were previously employed in the gene inactivation studies of $m p p G$ and $m p p E$ (Balakrishnan et al. 2017a, 2017b). The two fragments were cloned such that they flanked a $3.9 \mathrm{~kb}$ hygromycin resistance cassette (hyg) in pCAMBIA 1300 (GenBank accession no. AF234296) through a three fragment ligation using an InFusion cloning method (Clontech, Mountain View, CA, USA), generating pBIJ19 (Fig. 2A). The primer pair hyg-F/Hyg-mppE$\mathrm{R}$ was used to amplify hyg from pUR5750, which is a pBIN19 derivative containing hyg from pAN7.1 (de Groot et al. 1998). The inactivation construct pBIJ20 was introduced into M. purpureus through an Agrobacterium-mediated transformation as previously described (Michielse et al. 2008).

\section{Chemical analyses}

Thin layer chromatography (TLC) was performed on silica gel 60 $\mathrm{F}_{254}$ TLC plates (Merck, Darmstadt, Germany), which were developed with a mixture of n-hexane, ethyl acetate and formic acid (24:18:1). Ultraviolet (UV)-visible absorption spectra were collected with a Cary50 spectrophotometer (Varian, Palo Alto, CA, USA). HPLC analysis was performed on a ProStar system (Varian) with a GeminiC-18 column $(150 \times 3.0 \mathrm{~mm}, 5.0 \mathrm{~mm}$; Phenomenex, Torrance, CA, USA) and the elution was monitored at $280 \mathrm{~nm}$. The mobile phase consisted of water (A) and acetonitrile (B). The flow rate was maintained at $0.5 \mathrm{~mL} / \mathrm{min}$. The system was run with the following gradient program: $90 \% \mathrm{~A}$ for $5 \mathrm{~min}$, from $90 \%$ A to $100 \%$ B over $20 \mathrm{~min}$, then maintained at $100 \%$ B for $20 \mathrm{~min}$.

\section{Results}

Targeted deletion of $m p p D, m p p E$ and $m p p G$ in $M$. purpureus In the MAz biosynthetic gene cluster, $m p p D, m p p E$ and $m p p G$ are located between $m p p C$ and $m p p R 2$ (Fig. 2A). We have previously generated $\triangle m p p E$ and $\triangle m p p G$ mutant via hyg gene replacement (Balakrishnan et al. 2017a; 2017b). We attempted to generate an $m p p E$ and $m p p G$ double mutant by using revised versions of the mppE and $m p p G$ inactivation plasmids that contained an nptII gene, as was done in the citrinin biosynthetic gene study (Balakrishnan et al. 2016). However, we failed to obtain a transformant in several trials. The proximity of the two genes may have been a hurdle for the double inactivation. As an alternative 


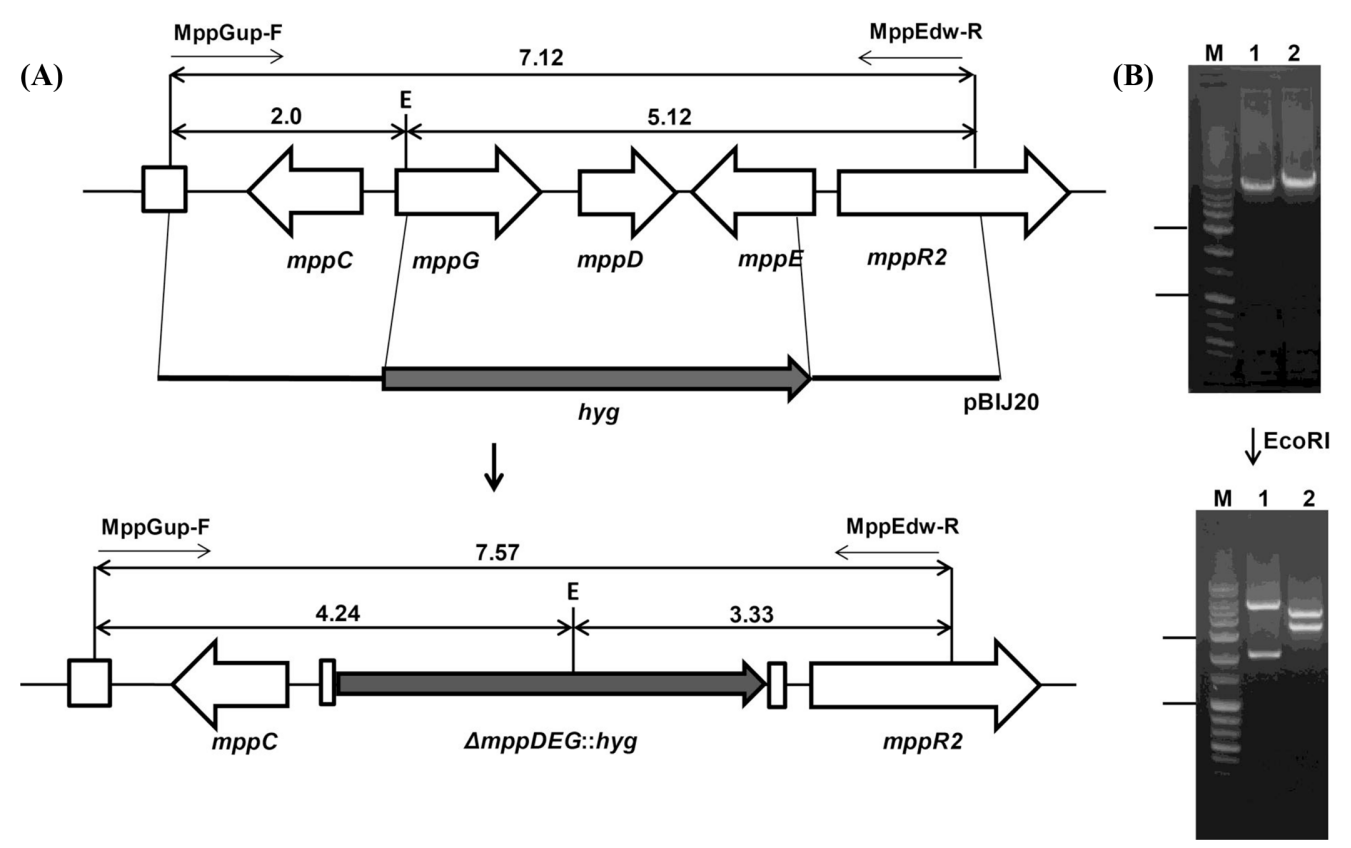

Fig. 2 Gene inactivation scheme for $m p p D, m p p E$ and $m p p G$ ( $m p p D E G$ ) in $M$. purpureus. (A) EcoRI restriction maps of the DNA region flanking $m p p D, m p p E$ and $m p p G$ in the WT strain, pBIJ20 and the resulting $\triangle m p p D E G$ mutant. (B) Analytical PCR using DNA from the WT (1) and the $\triangle m p p D E G$ mutant (2) strains with the primer pair MppGup-F and MppEdw-R. M indicates the DNA molecular weight marker with sizes of 0.1, 0.2, $0.3,0.4,0.5,0.6,0.8,1.0,1.5,2.0,3.0,4.0,5.0,6.0,8.0$ and $10.0 \mathrm{~kb}$ (from bottom to top). The 1.0 and $3.0 \mathrm{~kb}$ fragments are highlighted with bars

approach, we deleted a $3.4 \mathrm{~kb}$ region encompassing $m p p D$, $m p p E$ and $m p p G(m p p D E G)$ in $M$. purpureus (Fig. 2A). The inactivation construct pBIJ20 was designed to delete the $m p p D E G$ region and replace it with hyg through a double crossover. The primer pair MppGup-F/MppEdw-R is predicted to yield 7.1 and $7.6 \mathrm{~kb}$ PCR products for the wild-type (WT) and $m p p D E G$ knockout strains $(\triangle m p p D E G)$, respectively. In the PCR analysis, the $\triangle m p p D E G$ strain generated a band that was slightly larger than that from the WT strain (Fig. 2B). We also performed EcoRI digestions of the PCR products to distinguish between the WT and $\triangle m p p D E G$ strains (Fig. 2A). An EcoRI digestion of the PCR bands generated 3.3 and $4.2 \mathrm{~kb}$ sized bands for the $\triangle m p p D E G$ strain, which was clearly distinguishable from the WT digestion bands of 2.0 and $5.1 \mathrm{~kb}$ (Fig. 2B). Thus, it was confirmed that the ÄmppDEG mutant was successfully generated.

The $\triangle m p p D E G$ mutant retains the ability to produce MAz A comparison of MAz production between the $\triangle m p p D$ and $\triangle m p p D E G$ strains was expected to elucidate whether a functional redundancy of $m p p E$ and $m p p G$ gives rise to the residual yellow and orange MAz production in the $\triangle m p p E$ and $\triangle m p p G$ strains, respectively. An alternative scenario is that the residual production is not mediated by $m p p E$ nor by $m p p G$, but rather by nonspecific activities of unknown oxidoreductase(s) in $M$. purpureus. When grown in PDA (surface) and PDB (submerged) cultures, the $\triangle m p p D E G$ strain was found to retain MAz production as shown by the UV-Vis absorption spectra and TLC analysis (Fig. 3A and
C). UV-Vis absorption of the $\triangle m p p D$ and $\triangle m p p D E G$ extracts at the wavelengths relevant to MAz are compared with those of the WT extracts (Table 2). The $\triangle m p p D$ strain displayed approximately 10 to $20 \%$ of absorbance of that of the WT strain as reported previously (Balakrishnan et al. 2015). Unexpectedly, the absorbance values of the $\triangle m p p D E G$ extracts was comparable with or higher than those of the $\triangle m p p D$ extracts and an increased absorption at $500 \mathrm{~nm}$, indicative of red MAz, was observed in the $\triangle m p p D E G$ strain (Figs. 3A, C, and Table 2). Both orange and red MAz compounds have considerable absorption at approximately 400 $\mathrm{nm}$, as well as at $470-530 \mathrm{~nm}$. Thus, a visible absorption spectrum by itself is incapable of revealing the MAz profile in detail. A TLC analysis indicated the presence of Y-1 and Y-2 in PDA (Fig. $3 \mathrm{~B})$ and $\mathbf{O - 2}$ in PDB for the $\triangle m p p D E G$ strain (Fig. 3C). In the PDB culture, the cell growth of the $\triangle m p p D E G$ and $\triangle m p p D$ strains showed no difference with respect to dried cell weight $(6.2 \pm 0.2$ and $6.2 \pm 0.1$, respectively; the mean values \pm standard deviations were derived from triplet experiments). This indicates that $m p p E$ and $m p p G$ are not the only genes encoding enzymes for the final oxidoreduction steps in $\mathrm{MAz}$ biosynthesis in M. purpureus, although $m p p E$ and $m p p G$ remain the primary players.

\section{The $\triangle m p p D E G$ strain accumulates red MAz}

To determine the MAz profile of the $\triangle m p p D E G$ extract from the PDA culture, a reverse phase HPLC analysis was performed using the $\triangle m p p D$ and WT extracts as references (Fig. 4A). This HPLC analysis was expected to reveal the red MAz components from the 

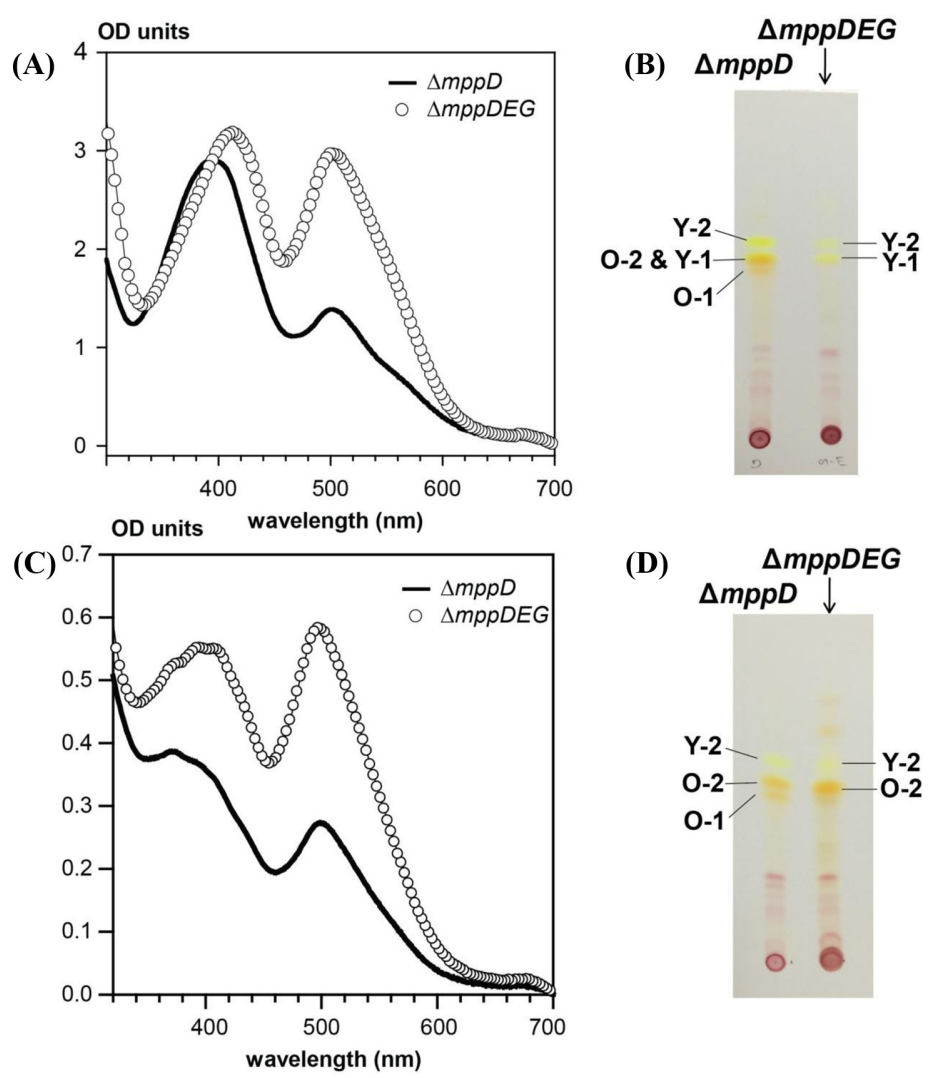

Fig. 3 UV-Vis absorption ( $\mathrm{A}$ and $\mathrm{C}$ ) and TLC (B and D) analysis of MAz production in the $\triangle m p p D$ and $\triangle m p p D E G$ mutants. The organic extract from PDA (A) and the mycelia pellets of the PDB cultures (C) were used to generate UV-Vis spectra with proper dilutions in methanol, and the data were converted to the corresponding values in the original culture volume. These values are indicated as OD units. The extracts of the $\triangle m p p D$ and $\triangle m p p D E G$ mutants are represented by a bold line and unfilled circles, respectively. TLC traces of the organic extracts from PDA (B) and the mycelia pellets of PDB cultures (D). Y-1 and $\mathbf{O - 2}$ overlap in this TLC condition

$\triangle m p p D E G$ mutant extract. A WT extract was included to validate that red $\mathrm{MAz}$ is enriched in the $\triangle m p p D E G$ strain. In the WT sample, Y-1, Y-2, O-1 and O-2 could be identified. Both Y-1 and Y-2 were detected in the $\triangle m p p D$ mutant sample, and their levels were significantly lower in the extract from the $\triangle m p p D E G$ strain. We previously estimated the content of Y-1 to be approximately 6 $\mathrm{mg}$ from each $50 \mathrm{~mL}$ PDA culture of the WT and $\triangle m p p G$ strains (Balakrishnan et al. 2017b). This information is used to estimate the content of Y-1 from the PDA extracts of the $\triangle m p p D$ and $\triangle m p p D E G$ mutants (Fig. 4A). The Y-1 contents of the $\triangle m p p D$ and $\triangle m p p D E G$ extracts were calculated to be approximately 3 and $0.3 \mathrm{mg}$, respectively, from each $50 \mathrm{~mL}$ PDA culture. This indicates that a reduction in UV-Vis absorption of the $\triangle m p p D$ extract from the PDA culture, compared to those of the WT strain (Table 2), resulted from a limitation in orange MAz production. In peak integration values in the HPLC chromatograms, the contents of Y-1 and Y-2 were found comparable for both the $\triangle m p p D$ and $\triangle m p p D E G$ extracts. As expected, the $\triangle m p p D E G$ strain accumulated two fast eluting compounds, designated as R-1 and R-2. It seemed reasonable to hypothesize that these two compounds were red MAz, and an LC-MS analysis of the HPLC eluate supported that they were the ethanolamine derivatives of red MAz (Fig. 4B and C). Both R-1 and R-2 are the ethanolamine derivatives of O-1 and O-2, having molecular formulas of $\mathrm{C}_{23} \mathrm{H}_{27} \mathrm{NO}_{5}$ and $\mathrm{C}_{25} \mathrm{H}_{31} \mathrm{NO}_{5}$, respectively. The positive ion masses of $\mathbf{R}-\mathbf{1}$ and $\mathbf{R}-\mathbf{2}$ are predicted to be $398.1962[\mathrm{M}+\mathrm{H}]^{+}$and $420.1781[\mathrm{M}+\mathrm{Na}]^{+}$; and 426.2275 $[\mathrm{M}+\mathrm{H}]^{+}$and $448.2094[\mathrm{M}+\mathrm{Na}]^{+}$, respectively. The Orbitrap mass analysis yielded $\mathrm{m} / \mathrm{z}$ values of 398.1952 and 420.1767; and 426.2272 and 448.2087 for R-1 and R-2, respectively, supporting their identities. R-2 was previously reported, named PP-R in a Penicillium species (Ogihara et al. 2001) but there is no report on R-1. Although R-1 has not been reported, its occurrence in the extract is not surprising that the formation of red MAz is nonenzymatic process. The LC-MS analysis of the $\triangle m p p D E G$ extract confirmed the presence of Y-1, Y-2 and $\mathbf{O - 2}$ in addition to R-1 and R-2 (data not shown). A peak that eluted between Y-1 and Y2 were not reduced in the $\triangle m p p D E G$ sample, compared to the $\triangle m p p D$ sample, and the LC-MS analysis determined this peak to be MC-3, which is an MAz derivative that accumulates in the $\triangle m p p C$ mutant (Bijinu et al. 2014). It is worthwhile to note that the proposed biosynthetic rout for MC-3 involves neither $m p p E$ nor $m p p G$ (Fig. 1). 
(A)
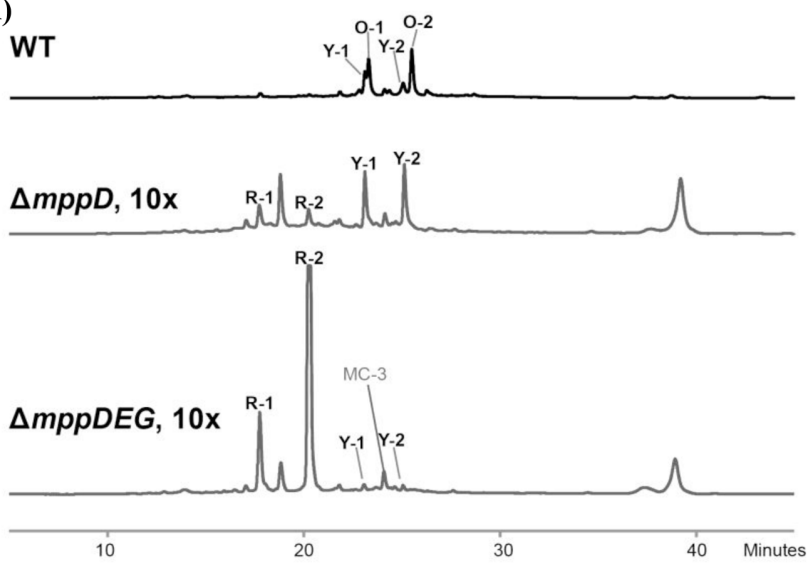

(B) R-1
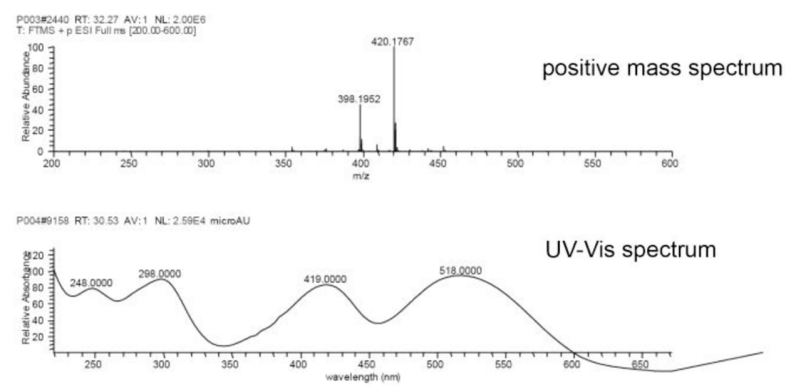

(C) R-2
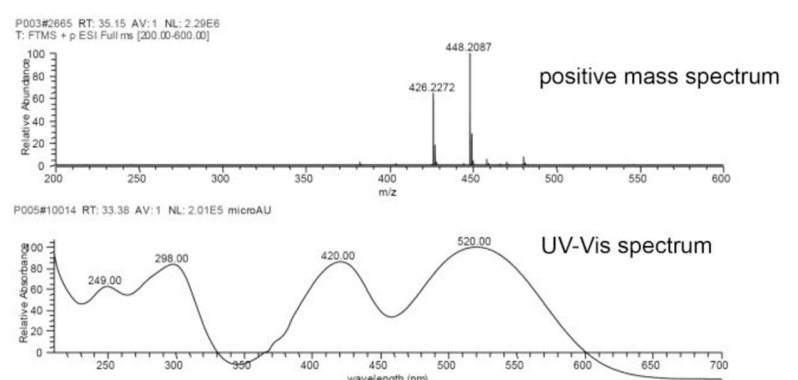

Fig. 4 HPLC analysis of MAz content in PDA culture extracts of the $\triangle m p p D$ and $\triangle m p p D E G$ mutants compared with that of the WT strain. (A) The extracts of the $\triangle m p p D$ and $\triangle m p p D E G$ mutants are concentrated tenfold compared to that of the WT strain and are indicated as $10 x$. The HPLC traces were monitored at $280 \mathrm{~nm}$ and are drawn to the same scale. The HPLC eluate of R-1 (B) and R-2 (C) were subjected to LC-MS analysis by positive electrospray ionization for determination of the molecular formulas and UV-Vis absorption spectra

\section{Discussion}

In this study, we generated a $\triangle m p p D E G$ mutant and compared its MAz production profile with an $\triangle m p p D$ mutant to access how the loss of both $m p p E$ and $m p p G$ affects MAz production. We found that the ability to synthesize MAz was retained despite the loss of both $m p p E$ and $m p p G$, although the MAz profiles of the $\triangle m p p D E G$ and $\triangle m p p D$ strains differed somewhat (Figs. 3 and 4). We could not deduce how these trivial differences occurred in the
Table 2 UV-Vis absorption of the $\triangle m p p D$ and $\triangle m p p D E G$ extracts at the wavelengths relevant to MAz are shown in comparison to those of the WT extracts. UV-Vis spectra were generated with proper dilutions in methanol, and the data were converted to the corresponding values in the original culture volume

\begin{tabular}{cccccc}
\hline \hline \multirow{2}{*}{$\begin{array}{c}\text { culture } \\
\text { medium }\end{array}$} & \multirow{2}{*}{ strain } & \multicolumn{4}{c}{ Absorbance at the given wavelength } \\
\cline { 3 - 6 } & & $390 \mathrm{~nm}$ & $410 \mathrm{~nm}$ & $470 \mathrm{~nm}$ & $500 \mathrm{~nm}$ \\
\hline \multirow{3}{*}{ PDA } & WT & 37 & 36 & 19 & 17 \\
& $\Delta m p p D$ & 2.9 & 2.8 & 1.1 & 1.4 \\
& $\Delta m p p D E G$ & 2.7 & 3.2 & 2.0 & 3.0 \\
\hline \multirow{4}{*}{ PDB } & WT & 19 & 19 & 14 & 12 \\
& $\Delta m p p D$ & 0.38 & 0.34 & 0.20 & 0.27 \\
& $\Delta m p D D E G$ & 0.55 & 0.57 & 0.44 & 0.59 \\
\hline
\end{tabular}

present study. These results led us to conclude that in $M$. purpureus, MAz production consists of major and minor pathways, where the former employs $m p p E$ or $m p p G$ while the latter does not. At present, it is impossible to deduce which genes are involved in the minor MAz biosynthetic routes. A notable feature of the $\triangle m p p D E G$ strain is the accumulation of red MAz derivatives (Fig. 4). MAz production levels in the $\triangle m p p D$ and $\triangle m p p D E G$ strains were much lower than the WT strain, but the relative levels of R-1 and R-2 were enhanced. We have previously observed that R-1 and R-2 levels were enhanced in a $\triangle m p p E$ strain, which accumulated orange MAz (Balakrishnan et al. 2017a). For a clear comparison between chromatograms, we assayed the $\triangle m p p E$ extract together with comparable concentrations of the $\triangle m p p D$ and $\triangle m p p D E G$ extracts (Fig. 5). Half as much R-1 and R-2 was observed in $\triangle m p p D E G$ extract compared to that of the $\triangle m p p E$ strain, as approximated by HPLC peak intensities (Fig. 5). A high level of red MAz in the $\triangle m p p E$ strain was proposed to have resulted from an accumulation of orange MAz, with red MAz originating from orange MAz. The high level of red MAz in the $\triangle m p p D E G$ strain could not be explained by this interpretation. We therefore speculate that a slow turnover in orange $\mathrm{MAz}$ production may favor red MAz production in the $\triangle m p p D E G$ strain. The $\triangle m p p D$ mutant was also relatively enriched for red MAz, supporting this slow turnover scenario (Fig. 4). It could also be interpreted that a high level of orange MAz production in the $\triangle m p p E$ strain may exert a feedback inhibition on MppG during the later stages of growth, reducing the production turnover and favoring red MAz accumulation. However, we could not identify a mechanism of how a slow turnover favors red MAz production. One possible mechanism involves cellular membrane regeneration, which is discussed below. We could not exclude the possibility that red MAz can be generated in vivo by a path that is independent of orange MAz; the hypothetical common intermediate MAz-INT is directly converted into red MAz (Fig. 1).

It remains to be determined how red MAz ethanolamine derivatives were selectively produced by the non-enzymatic conversion of orange to red MAzs. It is difficult to accept that the intracellular level of ethanolamine is high enough that orange 


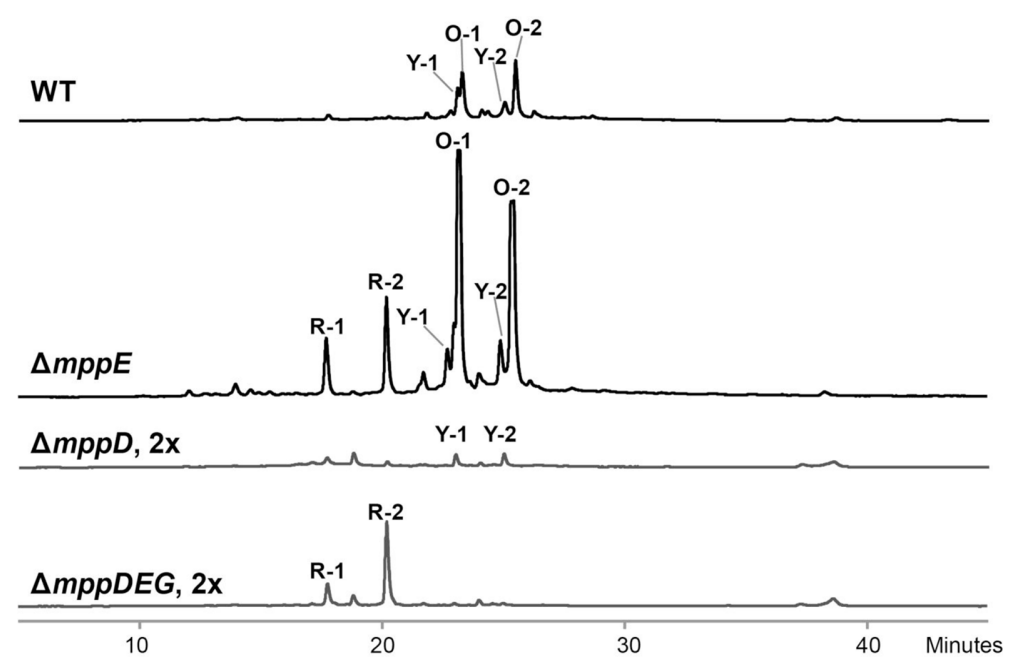

Fig. 5 HPLC chromatogram comparison of the PDA extracts from the WT, $\triangle m p p E, \triangle m p p D$ and $\triangle m p p D E G$ strains. HPLC traces were monitored at $280 \mathrm{~nm}$ and are drawn to the same scale. The extracts of the $\triangle m p p D$ and $\triangle m p p D E G$ mutants are twice as concentrated as that of the WT strain and are indicated as $2 x$

MAz preferentially reacts with it. When $\mathbf{0 - 4}$ was administrated to an albino mutant of $M$. purpureus ( $\triangle M p P K S 5)$ (Balakrishnan et al. 2013), the mycelial pellet readily exhibited a red color, but the red colorant was not extractible with methanol (data not shown). Trials to extract this red colorant that included additional steps, such as acidifying and boiling, did not make a difference. This implied that orange MAz production was not be directly correlated to red MAz production. All colored M. purpureus strains yield a non-extractable red color in the mycelial pellet. To the best of our knowledge, the chemical nature of this non-extractable red color has never been pursued, leaving in vivo production mechanism of red MAz undetermined. Because ethanolamine is a major amine moiety in biological phospholipids, we suggest that the in vivo formation of the red MAz preferentially occurs in the biological membrane where orange MAz localizes inside of or near a membrane being close to an ethanolamine moiety of phosphatidylethanolamine. During membrane remodeling, new membrane synthesis and old membrane hydrolysis during the mycelia growth may result in a release of red MAz ethanolamine derivatives.

In the present study, we generated an M. purpureus strain that selectively accumulates red MAz compounds by deleting three MAz biosynthetic genes ( $m p p D, m p p E$ and $m p p G$ ), although the mechanism by which these compounds accumulate remains undetermined. This strain could guide us to understand the underlying production dynamics of MAz and provide a favorable fermentation scheme to produce red MAz. However, whether the loss of $m p p D$ truly contributes to red MAz accumulation phenotype remains unknown.

Acknowledgment This research was supported by Basic Science Research Program through the National Research Foundation of Korea (NRF) funded by the Ministry of Education (NRF-2016R1D1A1B02009237).

\section{References}

Balakrishnan B, Chandran R, Park SH, Kwon HJ (2015) A new protein factor in the product formation of non-reducing fungal polyketide synthase with a $C$-terminus reductive domain. J Microbiol Biotechnol 25: 1648-1652

Balakrishnan B, Chandran R, Park SH, Kwon HJ (2016) Delineating citrinin biosynthesis: Ctn-ORF3 dioxygenase-mediated multi-step methyl oxidation precedes a reduction-mediated pyran ring cyclization. Bioorg Med Chem Lett 26: 392-396

Balakrishnan B, Chen CC, Pan TM, Kwon HJ (2014a) Mpp7 controls regioselective Knoevenagel condensation during the biosynthesis of Monascus azaphilone pigments. Tetrahedron Lett 55: 1640-1643

Balakrishnan B, Karki S, Chiu SH, Kim HJ, Suh JW, Nam B, Yoon YM, Chen CC, Kwon HJ (2013) Genetic localization and in vivo characterization of a Monascus azaphilone pigment biosynthetic gene cluster. Appl Microbiol Biotechnol 97: 6337-6345

Balakrishnan B, Kim HJ, Suh JW, Chen CC, Liu KH, Park SH, Kwon HJ (2014b) Monascus azaphilone pigment biosynthesis employs a dedicated fatty acid synthase for short chain fatty acyl moieties. J Kor Soc Appl Biol Chem 57: 191-196

Balakrishnan B, Park SH, Kwon HJ (2017a) A reductase gene $m p p E$ controls yellow component production in azaphilone polyketide pathway of Monascus. Biotechnol Lett 39: 163-169

Balakrishnan B, Park SH, Kwon HJ (2017b) Inactivation of the oxidase gene $m p p \mathrm{G}$ results in the selective loss of orange azaphilone pigments in Monascus purpureus. Appl Biol Chem doi:10.1007/s13765-017-0296-6

Bijinu B, Suh JW, Park SH, Kwon HJ (2014) Delineating Monascus azaphilone pigment biosynthesis: oxidoreductive modifications determine the ring cyclization pattern in azaphilone biosynthesis. RSC Adv 4: 59405-59408

Chen W, He Y, Zhou Y, Shao Y, Feng Y, Li M, Chen F (2015) Edible filamentous fungi from the species Monascus: early traditional fermentations, modern molecular biology, and future genomics. Com Rev Food Sci Food Safety 14: 555-567

Feng Y, Shao Y, Chen F (2012) Monascus pigments. Appl Microbiol Biotechnol 96: 1421-1440

Gao JM, Yang SX, Qin JC (2013) Azaphilones: chemistry and biology. Chem Rev 113: 4755-4811

de Groot MJ, Bundock P, Hooykaas PJ, Beijersbergen AG (1998) 
Agrobacterium tumefaciens-mediated transformation of filamentous fungi. Nat Biotechnol 16: 839-842

He Y, Cox RJ (2016) The molecular steps of citrinin biosynthesis in fungi. Chem Sci 7: 2119-2127

Lin CH, Lin TH, Pan TM (2017) Alleviation of metabolic syndrome by monascin and ankaflavin: the perspective of Monascus functional foods. Food Funct. doi:10.1039/c7fo00406k

Michielse CB, Hooykaas PJ, van den Hondel CA, Ram AF (2008) Agrobacterium-mediated transformation of the filamentous fungus Aspergillus awamori. Nat Protoc 3: 1671-1678

Nguyen T, Karl M, Santini A (2017) Red yeast rice. Foods 6: E19. doi:10.3390/foods6030019

Ogihara J, Kato J, Oishi K, Fujimoto Y (2001) PP-R, 7-(2-hydroxyethyl)monascorubramine, a red pigment produced in the mycelia of Penicillium sp. AZ. J Biosci Bioeng 91: 44-47
Patakova P (2013) Monascus secondary metabolites: production and biological activity. J Ind Microbiol Biotechnol 40: 169-181

Shi K, Chen G, Pistolozzi M, Xia F, Wu Z (2016) Improved analysis of Monascus pigments based on their $\mathrm{pH}$-sensitive Uv-Vis absorption and reactivity properties. Food Addit Contam Part A Chem Anal Control Expo Risk Assess. doi:10.1080/19440049.2016.1214289

Winter JM, Sato M, Sugimoto S, Chiou G, Garg NK, Tang Y, Watanabe K (2012) Identification and characterization of the chaetoviridin and chaetomugilin gene cluster in Chaetomium globosum reveal dual functions of an iterative highly-reducing polyketide synthase. J Am Chem Soc 134: 17900-17903

Zabala AO, Xu W, Chooi YH, Tang Y (2012) Characterizationof a silent azaphilone gene cluster from Aspergillus niger ATCC 1015 reveals a hydroxylation-mediated pyran-ring formation. Chem Biol 19: 10491059 